\title{
AUTOIMMUNE THYROIDITIS: RADIOLOGIST'S POINT OF VIEW
}

\author{
Rosairo S \\ Department of Radiology, Faculty of Medicine, University of Peradeniya \\ (Based on SNB Talwatta Memorial Oration delivered by the author in August 2015 at the \\ Annual Academic Sessions of Sri Lanka College of Radiologists)
}

Correspondence: Shanthini Rosairo <shanthinirosairo@yahoo.com>

Copyright: Shanthini Rosairo

\section{Introduction}

Thyroiditis is an inflammation of the thyroid gland. It encompasses many disorders which are classified by various schemes. Thyroiditis may be categorized as acute (suppurative), subacute (granulomatous or lymphocytic) and chronic (lymphocytic or invasive fibrous) thyroiditis. Each type has its own cause, clinical features, risk factors, diagnosis, consequences and prognosis. Autoimmune thyroiditis is the most common type of thyroiditis encountered and shows an increase in incidence in recent years in clinical practice. It includes Hashimoto's thyroiditis (chronic lymphocytic thyroiditis), painless postpartum thyroiditis and painless sporadic thyroiditis ${ }^{1}$. Painless postpartum thyroiditis and painless sporadic thyroiditis are subacute lymphocytic thyroiditis conditions which are indistinguishable, except by the relationship of the former to the pregnancy and postulated as subacute form of Hashimoto's thyroiditis. Hashimoto's thyroiditis is the most prevalent autoimmune disease of the thyroid gland $^{2}$. It is now over a century since the first description of the disease by the Japanese doctor Hakaru Hashimoto in 1912. Incidence and prevalence of autoimmune thyroiditis in Sri Lanka is on the increase ${ }^{8,9}$.

It is important to understand the aetiopathogenesis of autoimmune thyroiditis, which is still discussed and studied, for the diagnosis and management of the disease. Recent advances in thyroid imaging have considerably improved the diagnosis, treatment, follow-up and prognosis of thyroid diseases. This article will provide evidence for the cause of autoimmune thyroiditis and the 
consequences of this condition in the Sri Lankan setting which will help to tailor the interventions specifically targeting the population of Sri Lanka.

The thyroid gland plays an important role in regulating metabolic functions such as heart rate and cardiac output, lipid metabolism, heat regulation, and skeletal growth. Thyroid gland is a bi-lobed structure located in the lower neck. The left and right lobes are located immediately to the left and right of the trachea, respectively, and are connected anteriorly by a thin rim of thyroid tissue known as the isthmus. Thyroid gland is a highly vascular structure supplied by superior and inferior thyroid arteries.

\section{Imaging appearances of the normal thyroid gland}

High resolution ultrasonography (US) is the imaging modality of choice for the initial assessment of the thyroid gland. It allows a more accurate assessment of the size and morphology of the thyroid gland. The thyroid lobes are normally $4-6 \mathrm{~cm}$ in craniocaudal length and $1.3-1.8 \mathrm{~cm}$ in their anteroposterior and transverse dimensions; the isthmus normally has an anteroposterior thickness of up to $3 \mathrm{~mm}$. A preliminary study done in 2010 showed thyroid volumes of $6.41 \mathrm{ml}$ in females and $8.62 \mathrm{ml}$ in males, in a Sri Lankan population, which are lower than the west ${ }^{4}$. On US the normal thyroid tissue appears homogeneously echogenic with uniform echotexture. A normal thyroid gland is of higher echogenicity as compared with prethyroid muscles and is of similar or slightly higher echogenicity as compared with neighbouring submandibular glands. Color and spectral Doppler imaging are useful to evaluate vascularity of the thyroid gland $^{3}$. Normally, a low resistance flow with high peak systolic velocity (PSV) is detected in these vessels on spectral Doppler analysis. The normal PSV in the intraparenchymal arteries of thyroid ranges between $15-30 \mathrm{~cm} / \mathrm{s}$.

Computed tomography (CT) and magnetic resonance imaging (MRI) have an adjuvant role in the evaluation of thyroid diseases. On CT scan without contrast, the thyroid is homogeneous and mildly hyper attenuating relative to the surrounding neck musculature, with an attenuation of 80-100 HU. On MRI the thyroid is, slightly hyperintense on $\mathrm{T} 1-\mathrm{W}$ images and iso- to slightly hyper intense on $\mathrm{T} 2-\mathrm{W}$ images relative to the neck musculature. Contrast enhanced CT and MR imaging of thyroid shows homogeneous enhancement. MR imaging and $\mathrm{CT}$ provide precise anatomic information regarding the position of the thyroid relative to adjacent vascular and muscular structures but are less sensitive than US in evaluating intra thyroid lesions. On radionuclide scanning $(99 \mathrm{mTc}$ or $123 \mathrm{I}$, 131I), normal thyroid demonstrates symmetric uniform uptake. PET shows FDG uptake within the normal thyroid gland, similar to adjacent skeletal muscle. US remains the best modality for the evaluation of the thyroid.

\section{Prevalence of autoimmune thyroiditis}

The incidence of Hashimoto's thyroiditis in the west is approximately $0.3-1.5$ cases per 1,000 population per year ${ }^{1,5}$. Prevalence of subclinical disease is now known to be tenfold higher. Prevalence of painless postpartum thyroiditis ranges from 1.1 $21.1 \%{ }^{6}$. A Sri Lankan study conducted among school girls aged 11-17 years in 2001 revealed that the prevalence of serologically proven thyroiditis is $15.2 \%{ }^{7}$. Another study conducted in 2007 revealed that the goiter prevalence is $6.8 \%$ and $19.6 \%$ of goiters 
showed both serological and cytological evidence of autoimmune thyroiditis ${ }^{8}$. A recent study conducted by the author in Kandy district of Sri Lanka in 2014/2015 revealed prevalence of thyroiditis is $19 \%$ (unpublished data).

Incidence and prevalence of autoimmune thyroiditis in Sri Lanka is on the increase, probably as a consequence of universal salt iodization used to overcome the iodine deficiency disorders ${ }^{8,9}$. Increase in use of high resolution ultrasonography may be a confounding factor for higher detection rate of disease even before clinical presentation.

\section{Demography of autoimmune thyroiditis}

Hashimoto's thyroiditis is the most common type of thyroiditis with a peak age group of $30-50$ years and its incidence is estimated to be 10-15 times higher in females 5 . A research conducted by the author in a tertiary care hospital in central province of Sri Lanka showed that the age distribution of the thyroiditis patients ranged from 16-57 years $($ mean $=33.2$ years, $\mathrm{SD}=11.39$ years). A noteworthy observation in relation to the age is that the peak age distribution was reported in the 21-30 years age group, which is lower than the expected age group when compared with available international data ${ }^{10}$.

\section{Pathogenesis of autoimmune thyroiditis}

It is essential to have an understanding about the pathogenesis of the autoimmune thyroiditis and the pathological changes that occur in the thyroid gland prior to embark on interpretation of the imaging appearances.

Autoimmune diseases of the thyroid gland represent a spectrum of disorders which share the presence of lymphocytic infiltrate at variable intensities in the thyroid parenchyma and production of antithyroid antibodies. Pathogenesis of autoimmune thyroiditis is complex. Breakdown of immune tolerance by interplay of immunologic, genetic and environmental factors is an early event.

In chronic lymphocytic thyroiditis, anti thyroid immune response begins with activation of thyroid antigen specific $\mathrm{T}$ helper cells. Several theories are postulated for this activation ${ }^{1}$. The activated $\mathrm{T}$ helper cells induce $\mathrm{B}$ cells to secrete thyroid antibodies. Thyroid antibodies act against thyroid peroxidase (TPO) and thyroglobulin. Thyroid peroxidase antibodies are closely associated with overt thyroid dysfunction and it correlates with thyroid cell damage and lymphocytic infiltration by both $\mathrm{B}$ cells and cytotoxic $\mathrm{T}$ cells which is a common histological feature in autoimmune thyroiditis. Thyroglobulin antibodies are present less frequently, and their role is unclear. TPO antibodies are the hall mark of autoimmune thyroiditis and are present in almost all patients with Hashimoto's thyroiditis, in two-thirds of patients with postpartum thyroiditis, $50 \%$ of painless sporadic thyroiditis. It should be noted that $10-15 \%$ of the general population are also positive for TPO antibodies and that low titers are not specific for the diagnosis ${ }^{11}$.

Autoimmune thyroiditis is thought to be provoked in genetically susceptible individuals by environmental and endogenous triggers ${ }^{1,2}$. Genetic predisposition to development of the autoimmune thyroid diseases was established on the basis of the epidemiologic studies which indicated increased prevalence of such diseases in some families, especially in twins. According to current knowledge, appearance of Hashimoto's thyroiditis and 
postpartum thyroiditis in Caucasians are associated with some gene alleles ${ }^{1}$.

Endogenous factors such as female sex, rebound phenomenon in postpartum period, endocrine polyglandular disease and many other autoimmune diseases thought to be associated with autoimmune thyroiditis. Environmental factors such as excessive iodine intake, heavy metals, treatment with certain drugs (interferon $\alpha$, IL-2, lithium, amiodarone), infections, mainly viral and exposure to many chemicals such as polyaromatic hydrocarbons and phenyls are considered to provoke thyroid autoimmunity ${ }^{1}$.

Excess iodine intake is considered a causative factor for autoimmune thyroiditis. A study conducted in Sri Lanka has an increase of the incidence and prevalence of thyroid autoimmunity in Sri Lanka, probably as a byproduct of compulsory iodination of salt used to overcome highly prevalent hypothyroidism ${ }^{8}$. There is evidence to suggest that the iodine content of the salt obtained from several commercial outlets is inappropriate in our country. Fifty two percent of them reported to contain excess iodine, where as only $32 \%$ conformed with recommended concentration ${ }^{12}$.

Urinary iodine concentration is considered as a good surrogate marker of iodine intake as a large proportion of ingested iodine is excreted in urine. Another study conducted in Sri Lanka showed majority of the autoimmune thyroiditis patients as well as healthy controls had high urinary iodine concentration suggesting excessive consumption of iodine ${ }^{14}$. There was no statistically significant difference of the urinary iodine concentrations between the two groups. This leads to the postulation that there should be other triggers for the cause of autoimmune thyroiditis.

At present it is thought that autoimmune thyroiditis is provoked in genetically susceptible individuals by both environmental and endogenous triggers.

\section{Thyroid status in autoimmune thyroiditis}

Patients with thyroiditis may have hyperthyroid, euthyroid or hypothyroid status but most of the patients with thyroiditis present with hypothyroidism. Inflammatory destruction of the thyroid may lead to transient thyrotoxicosis as preformed thyroid hormones are released from the damaged gland. As thyroid hormone stores are depleted, there is often a progression through a period of euthyroidism to hypothyroidism.

\section{Diagnosis of autoimmune thyroiditis}

Diagnosis of autoimmune thyroiditis is based on the clinical, laboratory, sonographic and cytological diagnosis.

Clinically, the patients may present with symptoms of hypothyroidism and clinical examination may typically demonstrate only a diffuse goiter with lobulated surface and firm consistency. Patients with atrophic thyroiditis on contrary present with decreased glandular volume or progression of the disease with existing goiter.

The main laboratory marker is the presence of anti-thyroid serum antibodies. Antithyroglobulin antibodies ( $\mathrm{TgAb}$ ) are present in $60 \%$ of patients, while anti-thyroid microsomal antibodies are present in $95 \%$ of patients. Positive result for thyroid peroxidase antibody (TPOAb) is a subtly more sensitive marker for chronic autoimmune thyroiditis than a positive test for anti-thyroid microsomal antibodies ${ }^{15}$. 
TSH is done to assess the thyroid status.

Fine needle aspiration cytology (FNAC) is considered in patients where, despite clinical manifestations of chronic autoimmune thyroiditis and high anti-thyroid antibodies concentrations, a thyroid nodule is suspected or in diffuse fast growing goiter to rule out thyroid lymphoma.

\section{Ultrasonographic diagnosis}

Sonography has been a valuable tool in the diagnostic work-up of thyroid diseases and it has been shown to be of considerable value in diagnosis as well as for excluding the possibility of thyroiditis in patients with either goitre or deranged thyroid functional status.

Role of ultrasonography is debatable to date. Different investigators have shown conflicting results in different study populations and communities. Raber et al. in 2002, focused on the echo pattern of the thyroid gland and determined that abnormal thyroid ultrasound patterns were highly indicative of autoimmune thyroiditis and allows the detection of thyroid dysfunction with $96 \%$ probability ${ }^{16}$. Pedersen et.al supported this observation ${ }^{17}$. A study conducted in 2007 concluded that ultrasonography has a high specificity (90\%) but low sensitivity (35\%) for diagnosis of thyroiditis when compared with antithyroid peroxidase antibodies $^{18}$. In a study conducted at a tertiary care hospital in Sri Lanka by the author to evaluate of the role of ultrasonography in the diagnosis of autoimmune thyroiditis, it was shown that the sensitivity of ultrasonography in diagnosing thyroiditis was $89.47 \%$ while the specificity was $96.3 \%$. Its positive predictive value was $94.4 \%{ }^{10}$.
The typical normal thyroid parenchyma has high echogenecity due to follicular structure. In autoimmune thyroiditis both lymphocytic infiltration and disruption of normal tissue architecture cause a reduction in thyroid echogenecity. Thyroid hypoechogenicity is currently viewed as an early sign of thyroid autoimmunity. In cases of hypoechogenic thyroid gland with echogenic trabecula crossing the parenchyma, there is no doubt, that it is a classic case of thyroiditis and the diagnosis is confirmed. Presence of multiple discrete hypoechoic micronodules (1-6 mm size) is strongly suggestive of chronic thyroiditis. Parenchyma intermixed with focal areas mimicking nodules formation present throughout the parenchyma is a common finding in thyroiditis. These are compatible with pseudonodular areas due to lymphocytic infiltrates. Colour Doppler can be used to characterize the above nodules. It is recommended to describe it as an ill-defined hypoechoic area than a nodule. On the contrary if any nodule is identified within a gland which is affected by thyroiditis it should be deeply studied looking for the possibility of malignancy and further evaluated with $\mathrm{FNAC}^{19,20,21,22}$.

The features considered to suggest malignancy in a thyroid nodule are hypoechogenicity, solid consistency, greater nodular height than the width, presence of microcalcifications, absence of a peripheral halo or presence of an interrupted halo, presence of intranodular vascularity and presence of peripheral vascularity ${ }^{23,24}$. A study conducted by the author to assess the validity of ultrasonography in identifying malignant nodules showed that though single ultrasound characteristics aid in the diagnosis of malignant thyroid nodules, combination of characteristics considerably improve the diagnostic accuracy ${ }^{25}$. 
With colour Doppler, thyroiditis shows increased vascularity than the typical pattern seen in the normal thyroid gland. This finding is used in the evaluation of the gland with minimal textural alteration. Spectral pattern of the flow of the thyroid arteries show flow velocities within normal limits $(\leq$ $40 \mathrm{~cm} / \mathrm{sec}$ ) which allows the differentiation from Graves disease $(>50 \mathrm{~cm} / \mathrm{sec})$.

Ultrasound elastography is a relatively advanced sonographic technique that assesses the stiffness of tissues by measuring the degree of distortion under external pressure. Thyroid gland elastography is used to study hardness or elasticity of the thyroid nodule to differentiate malignant from benign lesions. A benign nodule is softer and deforms more easily, whereas the malignant nodule is harder and deforms less when compressed by ultrasound probe. Contrast-enhanced ultrasound (CE-US) is another technique that helps in characterizing a thyroid nodule. On CE-US, enhancement patterns are different in benign and malignant lesions. Ring enhancement is predictive of benign lesions, whereas heterogeneous enhancement favours malignant lesions. However, overlapping findings seem to limit the potential of this technique in the characterization of thyroid nodules ${ }^{3}$.

\section{Natural course and other metabolic co- morbidities of autoimmune thyroiditis}

Subacute thyroiditis may resolve without any serious sequel, but Hashimoto's thyroiditis is usually destructive, leading to eventual parenchymal fibrosis and hypothyroidism. Therefore, it is important to follow up the patients with thyroiditis as they may require supplement of thyroxine depending on the thyroid status.
Several authors have demonstrated an increased risk of thyroid carcinoma in the patients with thyroiditis owing to the intense mitosis and cellularity of the gland stimulated by TSH and due to the lymphocytic infiltrate leading to cell destruction. Also thyroid lymphoma may be preceded by chronic thyroiditis. Thyroid lymphoma and papillary carcinoma though uncommon complications of autoimmune thyroiditis need to be diagnosed early if complete cure is aimed at, highlighting the importance of close follow up of patients with autoimmune thyroiditis ${ }^{26,27}$.

To assess the metabolic co-morbidities of autoimmune thyroiditis, a study was conducted by the author to assess the effect of thyroiditis on antioxidant status and lipid oxidation. Total antioxidant capacity and protein thiol concentrations were assessed in a group of thyroiditis patients and apparently healthy controls. This study showed similar protein thiol and total antioxidant values in both patients and control groups, indicating that the antioxidant status in both the groups are not significantly different ${ }^{14}$. Thiobarbituric acid reactive substance (TBARS) is generated during oxidation of lipids by free radicals. TBARS levels of thyroiditis patients and apparently healthy controls were compared. The study revealed evidence of lipid oxidation in thyroiditis patients which may have a role in the pathogenesis of atherosclerosis ${ }^{28,29,30}$.

\section{Conclusion}

Prevalence of autoimmune thyroiditis has increased in our country while the affected age group is becoming younger. Most of the affected individuals develop hypothyroidism requiring thyroxine supplement and follow up. Ultrasonography has proved to be of 
considerable value in the early diagnosis of thyroiditis as well as to establish the absence of thyroiditis. Ultrasonography also plays a valuable role in the follow up and diagnosis of the complications of thyroiditis such as papillary carcinoma and lymphoma. Awareness and diagnosis of co-mobities is helpful in the management of the patients. Since the autoimmune thyroiditis is a chronic disease with no curative treatment, assessment of the postulated risk factors may help to control the disease. This article has explored some of the facts of autoimmune thyroiditis, which may help to combat this emerging problem in Sri Lanka.

\section{References}

1. Pearce EN, Farwell AP, Braverman LE. Thyroiditis. New England Journal of Medicine. 2003;348(26):2646-55.

2. Syrenicz, Hashimoto's disease - from theory to practice. Thyroid Research. 2013; 6 (Suppl 2):A60.

3. Chaudhary V, Bano S. Imaging of the thyroid: Recent advances. Indian journal of endocrinology and metabolism. 2012;16(3):371-6.

4. Fernando R, Bandara DM, De Silva A, Renuka MD, Atulugama N. Thyroid volume in a cohort of Sri Lankan patients: a preliminary report. Ceylon Medical Journal. 2010;55(3):92.

5. Vanderpump MP, Tunbridge WM, French JM et al. The incidence of thyroid disorders in the community: a twenty-year follow-up of the Whickham Survey. Clinical Endocrinology. 1995; 43(1):55-68.

6. Muller AF, Drexhage HA, Bregout A. Postpartum thyroiditis and autoimmune thyroiditis in women of child bearing age: recent insights and consequences for antenatal and postnatal care. Endocrine Review. 2001; 22(5): 605-30.

7. Premawardhana LD, Parkes AB, Smyth PP, Wijeyaratne CN, Jayasinghe A, De Silva DG, Lazarus JH. Increased prevalence of thyroglobulin antibodies in Sri Lankan schoolgirls--is iodine the cause?. European journal of endocrinology. 2000; 143(2):185-8.

8. Fernando RF, Chandrasinghe PC, Pathmeswaran AA.The prevalence of autoimmune thyroiditis after universal salt iodisation in Sri Lanka. Ceylon Medical Journal. 2012 ;57(3):116-9.

9. Samarawickrama MB, Perera BL. Thyroiditis: can we depend on the clinical features to diagnose? Galle Medical Journal. 2009;14(1):35-8.

10. Rosairo S, Dandeniya CL, Wettasinghe $\mathrm{MC}$, Ratnatunga $\mathrm{C}$ and Galketiya $\mathrm{KB}$. Evaluation of the role of ultrasonography in the diagnosis and follow up of autoimmune thyroiditis: Proceedings of the Peradeniya University Research Sessions, 2010; 15(1):116-118.

11. Prummel MF, Wierasinga WM, Thyroid peroxidase auto antibodies in euthyroid subjects. Autoimmune endocrine disorders. 2005; 19(1): 1-15.

12. Kumarasiri JP, Fernandopulle BM, Lankathillake MA. Iodine content of commercially available iodised salt in the Sri Lankan market. The Ceylon Medical Journal. 1998; 43(2):84-7.

13. Camargo RY, Tomimori EK, Neves SC, Rubio IG, Galrão AL, Knobel M, Medeiros-Neto G. Thyroid and the environment: exposure to excessive nutritional iodine increases the prevalence of thyroid disorders in Sao 
Paulo, Brazil. European Journal of Endocrinology. 2008; 159(3):293-9.

14. Samarakoon E, Sivakanesan R, Rosairo S. Urinary iodine excretion and antioxidant status in patients with thyroiditis. Proceedings of the International conference on multidisciplinary approaches. 2014.

15. Dayan CM, Daniels GH. Chronic autoimmune thyroiditis. New England journal of Medicine 1996; 335(2):99107.

16. Raber W, Gessel A, Nowotny P, Vierhapper H. Thyroid Ultrasound Versus Antithyroid Peroxidase Antibody Determination: A Cohort Study of Four Hundred Fifty-One Subjects. Thyroid.2002; 12(8): 725-31.

17. Pedersen OM, Aardal NP, Larssen TB, Varhauq JE, Myking O, Vik-Mo H. Value of Ultrasonography in Predicting Autoimmune Thyroid Disease. Thyroid. 2000; 10(3): 251-259.

18. Tabur Suzan MD, YasAhmet O MD, Koylu Ahmet O MD, Sabuncu Tevfik MD..Sensitivity and Specificity of Ultrasonography in Detecting Thyroiditis. The Endocrinologist. 2007; 17(1):5-6.

19. Marwaha RK, Tandon N, Kanwar R et al. Evaluation of the Role of Ultrasonography in Diagnosis of Autoimmune Thyroiditis in Goitrous Children. Indian Pediatrics.2008; 45:279-284.

20. Danilo Bianchini Höfling, Giovanni Guido Cerri, Adriana Gonçalves Juliano, Suemi Marui, Maria Cristina Chammas. Value of thyroid echogenicity in the diagnosis of chronic autoimmune thyroiditis. Radiology Brasil. 2008;41(6):409-417.
21. Lauren Anderson, William D. Middleton, Sharlene A Teefey, Hashimoto Thyroiditis. Part I, Sonographic Analysis of the Nodular Form of Hashimoto Thyroiditis. American Journal of Roentgenology 2010; 195(1):208-215.

22. Daniel Smutek, Radim Sara, Peter Suchadra. Relation between quantitative description of ultrasonographic image and clinical and laboratory findings in lymphocytic thyroiditis. Endocrine Regulations. 2003; 37(3):181-7.

23. Won Jin Moon, Jung Hwan Baek, So Lyung Jung et al. Ultrasonography and the ultrasound-based management of Thyroid Nodules: Consensus Statement and Recommendations. Korean Journal of Radiolology. 2011; 12 (1):1-14.

24. Jenny K. Hoang, Wai Kit Lee, Michael Lee, Daryl Johnson, Stephen Farrel. US features of Thyroid Malignancy: Pearls and Pitfalls. Radiographics. 2007; 27(3):847-860.

25. Rosairo S, Ratnatunge NVI, Wettasinghe $\mathrm{MC}$.The validity of ultrasonographic characteristics in identification of malignant thyroid nodules: Proceedings of 12th Annual Academic Sessions of Sri Lanka College of Radiologists \& 7th SAARC Congress of Radiology.2013: 59.

26. Siriweera EH, Ratnatunga NV. Profile of Hashimoto's thyroiditis in Sri Lankans: is there an increased risk of ancillary pathologies in Hashimoto's thyroiditis? Journal of thyroid research. $2010 ; 10$. 
27. Lee JH, Kim Y, Choi JW, Kim YS. The association between papillary thyroid carcinoma and histologically proven Hashimoto's thyroiditis: a metaanalysis. European Journal of Endocrinology 2013; 168(3):34.

28. Dakhel Ghani Omran AlWatify.Oxidative Stress in Hypothyroidism. Journal of Babylon University/Pure and Applied Sciences. 2011; 19 (2): 444-449.

29. Samarakoon E, Sivakanesan R, Rosairo S. Serum concentration of thiobarbituric acid reactive substance in thyroiditis patients. Proceedings of the Postgraduate Institute of Science Research Congress Sri Lanka. 2014.

30. Baskol G, Atmaca H, Taniverdi F, Baskol M, Kocer D, Bayram F. Oxidative stress and enzymatic antioxidant status in patients with hypothyroidism before and after treatment. Expert Clinical Endocrinol Diabetes 2007; 115(8):522-6. 